\title{
Complete obstruction to flow in a three-way stopcock due to a manufacturing defect
}

\author{
Andrew D. Milne, MD • Paul A. Brousseau, BEd
}

Received: 29 August 2013/ Accepted: 9 September 2013/Published online: 27 September 2013

(c) Canadian Anesthesiologists' Society 2013

\section{To the Editor,}

Recently, when preparing our heated intravenous sets for intraoperative use, we detected a series of defective three-way stopcocks (MX5311L, Smiths Medical, Dublin, OH, USA) that caused complete obstruction to flow. Our typical heated fluid line setup includes a three-way stopcock placed between a set of heated tubing (Hotline ${ }^{\circledR}$ L-70) and a 30-in tubing extension connected to an intravenous cannula. Every morning, our anesthesia technicians routinely prepare batches of these sets for use throughout the day. Although these intravenous sets had been adequately de-aired and all the clamps were fully opened, we noticed that several were obstructed to passive gravity fed flow. Initially, our technicians were rejecting all three components and disposing of the entire setup. This disconcerting pattern was reported after several incidences, and a faulty set was kept for detailed examination. When troubleshooting a defective setup, the heated fluid line and extension components were observed to deliver full unrestricted flow when the stopcock was removed, thus implicating the three-way stopcock as the cause of the obstruction. Close examination of the white valve within

A. D. Milne, MD $(\bowtie) \cdot$ P. A. Brousseau, BEd

Department of Anesthesiology, Pain Management and

Perioperative Medicine, Dalhousie University, Halifax, NS, Canada

e-mail: admilne@dal.ca

A. D. Milne, MD

School of Biomedical Engineering, Dalhousie University,

Halifax, NS, Canada

P. A. Brousseau, BEd

QEII Health Sciences Centre, Dalhousie University, Halifax, NS,

Canada the clear plastic housing showed an incomplete perforation of the "through hole" in the valve body that blocked the passage of fluid (Figure). We immediately notified our staff, Biomedical Engineering, our Quality Control Department, and the local supplier and then performed a full search of our inventory to isolate any other defective units and locate a sample within its packaging to identify the lot number. One more defective three-way stopcock was found during this search, but unfortunately, our technicians had already disposed of its packaging. Despite an exhaustive search, we were initially unable to find a defective unit within its packaging to identify the specific lot number. Our inventory at the time was found to contain 30 different lot numbers, obscuring our search for the defective lot.

Our institution typically uses up to 30-40 of these units per day. We estimate that approximately 12 sets were thrown out over a one- to two-week period before the defect was discovered and that two of the units may have turned up in the operating room in emergent situations. In one case, a hot line and stopcock setup was assembled in the operating room for connection to a new intravenous cannula that was urgently placed when a pulmonary artery bleed occurred. Despite troubleshooting all the clamps and connections and applying pressure to the intravenous bag, obstruction of flow through the intravenous cannula persisted, initially casting doubt on the placement of the intravenous cannula. A second setup was quickly substituted and the obstruction immediately resolved. In the second suspected case, a stopcock was inserted in-line within an existing intravenous line during an acute bleed from the liver. The previously functioning intravenous line immediately stopped flowing with the stopcock insertion and then flowed smoothly after its removal. Both of these situations occurred under acute situations when the equipment was set up within the 


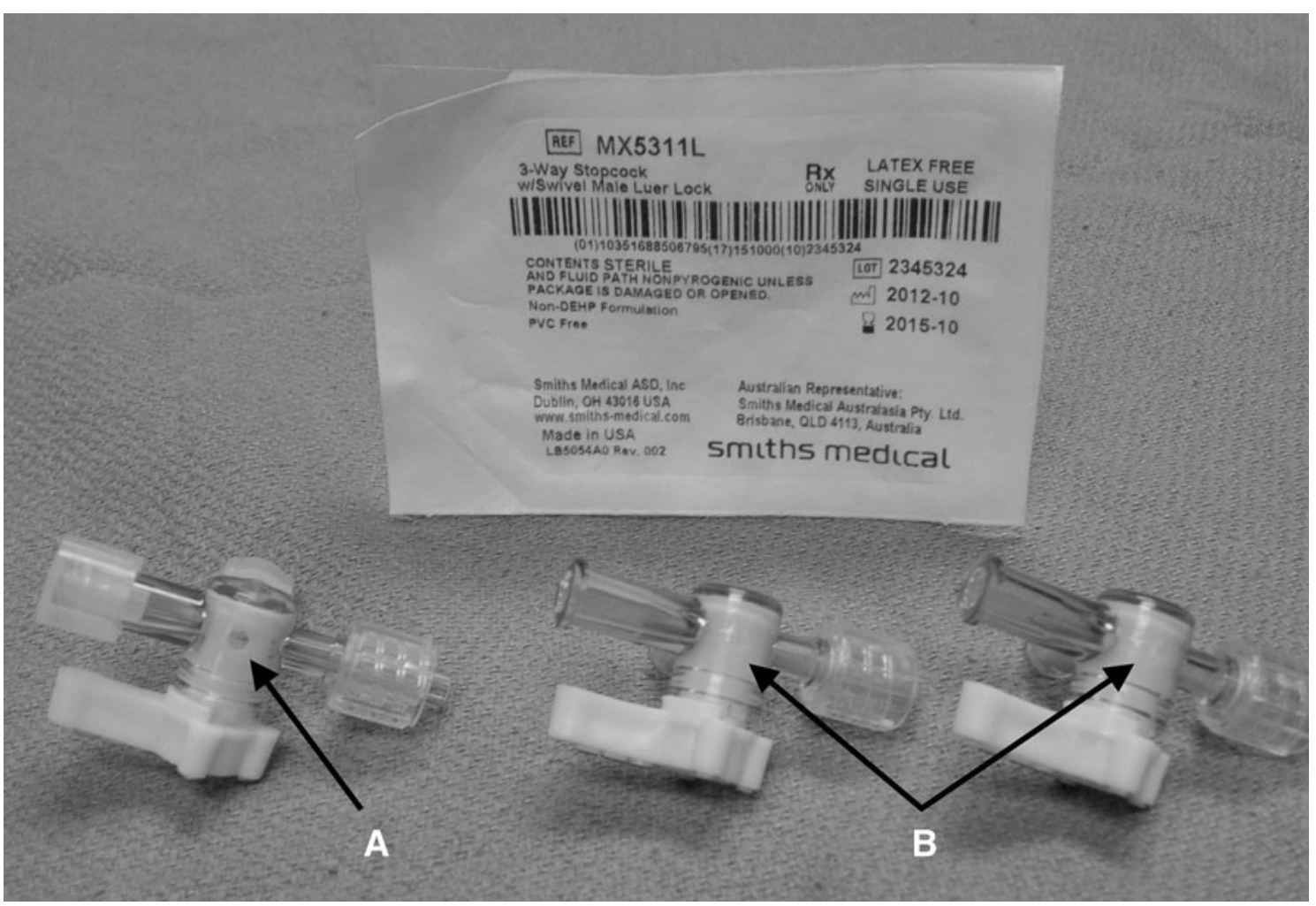

Figure Photograph of three-way stopcocks with the valve body rotated $90^{\circ}$ clockwise to show the defect. The normal sample on the left (A) has a complete "through hole" to allow passage of fluid, whereas the two defective samples to the right (B) have an incomplete

operating theatre (rather than preoperatively) and prior to our knowledge of the defect. At the time, neither set was kept to verify the cause of the blockage.

Similar three-way valves have been reported to fail after exposure to propofol infusions; ${ }^{1}$ however, in this case, the units were faulty before usage. Response from the manufacturer indicates that the defect was identified by the number "39" stamped on side of the white valve body and tracked through the maintenance records at their facility, and the inventory was isolated and inspected for further problems. We have notified Health Canada of this issue through the Canadian Medical Devices Sentinel Network (http://www.hc-sc.gc.ca/contact/dhp-mps/hpfb- or absent perforation on the inflow side of the valve body. The packaging shown is for identification purposes only and does not represent the defective lot

dgpsa/cmdsnet-resscmm-eng.php). This case highlights the importance of testing equipment before use and maintaining vigilance when using disposable equipment in the operating room. (Addendum - at the time of acceptance for publication a defective unit with the lot number 2300234 was subsequently identified.)

Conflicts of interest None declared.

\section{Reference}

1. Puri GD, Singh KP. Damage to three-way valves by a clear propofol formulation. Anesth Analg 2004; 98: 1193. 